\title{
Estudio exploratorio de la microbiota edáfica en el Centro de Investigación y Educación Ambiental La Tribuna, Neiva-Huila
}

\section{Exploratory study of the edaphic microbiota at the La Tribuna Environmental Education and Research Center, Neiva-Huila}

\author{
Sonia Echeverry Hernández ${ }^{*}$, Carlos Alberto Méndez Puentes²
}

\section{Resumen}

En general, los ecosistemas de bosque seco tropical no han sido suficientemente estudiados en comparación con otras zonas de vida, de allí se deriva la importancia de realizar este estudio en el Centro de Investigación y Educación Ambiental La Tribuna, asentamiento antiguo de explotación petrolera, con el objeto de establecer las posibles diferencias significativas entre poblaciones microbianas de algunos grupos funcionales del ciclo del carbono y del nitrógeno, heterótrofas totales y hongos por parcela y períodos pluviométricos. Las diferencias significativas observadas en este estudio se presentaron entre las poblaciones de amilolíticos y fijadores de nitrógeno como consecuencia de la temporada de lluvias o de la temporada seca; en las diferentes parcelas, los bacilos grampositivos esporulados fueron los microorganismos más abundantes presentando frecuencias de aparición entre común y muy común.

Palabras clave: Bosque seco tropical; ciclos biogeoquímicos; densidad poblacional; condiciones ambientales

\begin{abstract}
In general, tropical dry forest ecosystems have not been sufficiently studied compared to other life zones; from there, the importance of carrying out this study in the Center for Research and Environmental Education La Tribuna, an old oil exploration settlement, in order to establish the possible significant differences between microbial populations of some functional groups of the carbon and nitrogen cycle, total heterotrophs and fungi per plot and rainfall periods. The significant differences observed in this study were between the amylolitics and nitrogen fixative populations as a consequence of the rainy season or the dry season; in the different plots, the sporulated gram-positive bacilli were the most abundant microorganisms, presenting frequencies of appearance between common and very common.
\end{abstract}

Key words: Tropical dry forest; biogeochemical cycles; population density; environmental conditions

\footnotetext{
1* Universidad Surcolombiana, Profesora asociada. sonia.echeverry@ usco.edu.co, M.Sc Microbiología *(Autor para correspondencia)

${ }^{2}$ Secretaría de Educación del Huila. carlosalbertomendezpuentes@ gmail.com, Licenciado en Ciencias Naturales: Física, Química y Biología
} 


\section{INTRODUCCIÓN}

De acuerdo con el Instituto de Investigación de Recursos Biológicos Alexander Von Humboldt, el Bosque Seco Tropical (BST) es uno de los ecosistemas más amenazados a nivel mundial y el menos estudiado, pese a su trascendental importancia en la estabilidad de los suelos, el control de la erosión, la regulación hídrica y la provisión de alimentos y madera. Dada las características de doble estacionalidad pluviométrica de este ecosistema: lluvias y sequías prolongadas, muchas especies tanto vegetales, animales y microbianas han debido adaptarse desde su morfología y físiología hasta su comportamiento, con el fin de asegurar su supervivencia (Pizano y García, 2014). En Colombia, el BST comprende seis zonas geográficamente diferenciadas: el valle del río Patía en el sur del valle geográfico del Cauca, el valle del río Cauca, el alto y medio valle del río Magdalena, Santander y Norte de Santander, la costa Caribe, y la Orinoquía.

Sobre la microcuenca de la quebrada El Neme, (afluente de la cuenca baja del río Baché), al norte del departamento del Huila, en estribaciones de la cordillera central, margen izquierda del río Magdalena, se encuentra ubicada la finca de Ecopetrol conocida como "Centro de Investigación y Educación Ambiental, La Tribuna"; la cual hace parte del campo petrolero de San Francisco, dentro de la jurisdicción del municipio de Neiva. Desde el punto de vista geológico-estructural, este Centro pertenece a un anticlinal asimétrico y fallado conformado por sedimentos del Pre-cretáceo, el Cretáceo y el Mioceno (Vargas C et al, citado por Olaya y Gutiérrez, 2014).

Este Centro tiene un área aproximada de 150 ha, una altitud de 700 m.s.n.m., una temperatura promedio de $24^{\circ} \mathrm{C}$ y una precipitación anual que supera los $1200 \mathrm{~mm}$, distribuidos en un comportamiento bimodal siendo los meses de marzo, abril, mayo, octubre, noviembre y diciembre de temporada lluviosa y los meses de enero, febrero, junio, julio, agosto y septiembre de temporada seca; por lo anterior, La Tribuna corresponde a la zona de vida de bosque seco tropical (bs-T) de acuerdo con el sistema bioclimatológico de Holdridge. En este lugar se han desarrollado actividades de ecoturismo, de educación ambiental y de investigación, dentro de las que se destacan los estudios en flora nativa y fauna silvestre, así como los estudios hidrográficos, geológicos y de suelos (Olaya y Gutiérrez, 2014). En el año 2014, el Instituto de Investigación de Recursos Biológicos Alexander Von Humboldt, reportó la pérdida de más de la mitad del área que originalmente correspondía a BST en el país conduciéndolo a la desertificación, razón de más para que tanto autoridades ambientales, gubernamentales y civiles establezcan modelos de conservación y restauración de este ecosistema que garantice la vida de miles de especies vegetales y animales que se encuentran amenazadas y en vía de extinción.

Por tal motivo, en esta investigación se aislaron e identificaron mediante técnicas clásicas de Microbiología las poblaciones microbianas de algunos grupos funcionales de los ciclos del carbono y del nitrógeno, heterótrofas totales y hongos en distintas parcelas establecidas dentro del bosque y en las dos temporadas climatológicas del año: la lluviosa y la seca, con el fin de establecer la relación entre la distribución de los microorganismos en las diferentes parcelas y las condiciones del medio físico.

\section{MATERIALES Y MÉTODOS}

Zonas de muestreo: Se establecieron tres parcelas de 0,1 ha en tres zonas de La Tribuna (Figura 1), cada una de ellas con características tanto geológicas como agrológicas distintas: la parcela 1, ubicada en la parte alta de la microcuenca presenta suelos tipo antisol, de relieve fuerte (25-35\%) a muy fuerte (35-50\%), vegetación arbustiva y arbórea, con presencia de cauces y con influencia del asentamiento humano San Francisco, donde se realizan actividades de explotación petrolera (Olaya y Gutiérrez, 2014).

La parcela 2, ubicada en la parte media de la microcuenca, presenta rocas sedimentarias de formación tipo Villeta conformada, principalmente, por lodolitas carbonosas con niveles de calizas; los suelos se caracterizan por ser superficiales y moderadamente profundos con pendientes de relieve muy fuerte, entre el 35 y el $50 \%$ de inclinación. Los suelos superficiales se encuentran asociados a rocas de las formaciones Honda y Monserrate, 
con escaso horizonte A (HA) y el horizonte B (HB) localmente es de muy poco espesor; comúnmente en la superficie de estas formaciones geológicas, se presenta un suelo de tipo Regolítico (pedregoso) principalmente asociado a la formación Monserrate.

Los suelos moderadamente profundos se encuentran asociados a las rocas de la formación Villeta presentando los tres horizontes claramente distinguibles y con espesores de 0.30 a $1 \mathrm{~m}$; también presentan en su horizonte A una capa de humus generando buena cobertura vegetal. La vegetación es, principalmente, arbustiva y arbórea; se encuentran bosques protectores de cauces y malezas, y fauna; además, son abundantes los nacimientos de agua, saltos y cascadas lo que ha permitido clasificar esta zona como bosque secundario (Olaya y Gutiérrez, 2014).

La parcela 3, ubicada en la parte baja de la microcuenca presenta rocas de formación Honda representadas por arcillolitas de color rojo y crema de carácter semiplástico y granulométricamente con proporciones de arena (45\%), limo (30\%) y arcilla (25\%); localmente, se encuentran intercalaciones de limolitas en capas tabulares de estratificación media a fina y areniscas de granulometría de muy fina a conglomerática y niveles conglomeráticos en capas medias. El ambiente sedimentario que formó estos depósitos clásticos es de tipo lacustre donde reinaban los pantanos y lagos en una selva tropical húmeda. La edad de las rocas de la formación Honda presentes en La Tribuna corresponde al periodo del Mioceno antes del levantamiento de la Cordillera Oriental (Vargas, 2014).

Los suelos de esta parcela presentan relieve accidentado y pendientes que se encuentran entre el 12 y el $20 \%$. La vegetación característica comprende rastrojos, pastos naturales y enmalezados además de áreas erosionadas con poca vegetación, lo que hace que el uso potencial del suelo corresponda a la categoría de sistema silvoagrícola y silvopastoril (Olaya y Gutiérrez, 2014).

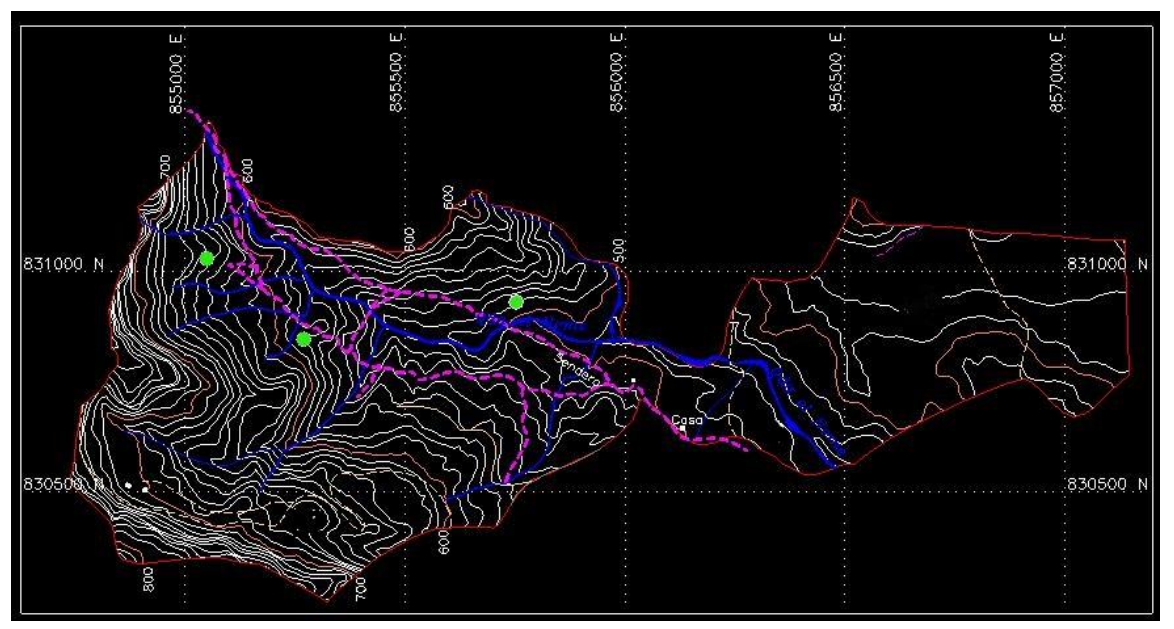

Figura 1. Área de estudio: en color verde se resalta el sitio donde se establecieron las parcelas

Toma de muestras: Los muestreos se realizaron en el periodo comprendido entre los meses de octubre del año 2013 y julio del año 2014, abarcando los meses de lluvias octubre y diciembre de 2013 y abril de 2014 (designados como M1, M2 y M3, respectivamente) y el mes de temporada seca julio de 2014 (designado como M4), dando como resultado una mayor recolección de muestras durante la temporada de lluvias en relación con la temporada seca. En cada parcela se realizaron dos transectos en diagonal con el fin de recolectar cinco submuestras: una por cada esquina y una del centro tomadas a una profundidad de $15 \mathrm{~cm}$ mediante barreno manual y descartando la vegetación superficial (Facultad de Ciencias Agropecuarias, Universidad Nacional de Córdoba, 2015). 
De la muestra final, compuesta de las cinco submuestras, se tomaron aproximadamente $100 \mathrm{~g}$, los cuales se depositaron en bolsas tipo zi-ploc rotuladas con los siguientes datos: número de parcela y fecha de recolección; las muestras fueron transportadas al laboratorio de Microbiología de la Universidad Surcolombiana y refrigeradas inmediatamente para su posterior análisis.

Procesamiento de muestras: de los $100 \mathrm{~g}$ de muestra por parcela se homogenizaron $10 \mathrm{~g}$ en $90 \mathrm{~mL}$ de agua peptonada estéril y a partir de ella se realizaron diluciones seriadas con un factor de dilución 1/10 hasta completar la dilución $10^{-6}$ (Valencia, 2010). $100 \mu \mathrm{L}$ de cada dilución se sembró por extensión en superficie y por duplicado en los siguientes medios de cultivo: agar tripticasa soya (ATS) para heterótrofas totales, medio de cultivo para amilolíticos (Pontecorcorvo et al, 1953), medio de cultivo de Burk para fijadores de nitrógeno que utilizan glucosa como fuente de carbono (Wilson y Knight, 1952), medio de cultivo Nfb para fijadores de nitrógeno que utilizan malato como fuente de carbono (Döbereiner y Day, 1976) y medio de Martin con estreptomicina para hongos totales.

Las placas se incubaron a $28^{\circ} \mathrm{C}$ durante 48 horas para el recuento de heterótrofos totales, durante 72 horas para fijadores de nitrógeno y durante 5 días para amilolíticos y hongos; los tamaños poblacionales se expresaron en UFC/g. Los morfotipos representativos se purificaron en ATS para su descripción morfológica; para la identificación de los microorganismos amilolíticos se tuvo en cuenta la formación de un halo alrededor de las colonias al revelarse con solución de iodo y para las bacterias fijadoras de nitrógeno, todas las colonias que se desarrollaron en los medios de cultivo se consideraron positivas; los hongos fueron identificados por descripción morfológica.

Descripción de morfotipos: La descripción morfológica de los cultivos bacterianos purificados se realizó teniendo en cuenta características como pigmentación, forma, elevación, borde y tamaño de las colonias; para la descripción de la morfología celular se empleó la tinción de Gram (Valencia, 2004).

Las colonias fúngicas se describieron teniendo en cuenta características como pigmentación, presencia de hifas aéreas, plegamientos, zonación, tamaño y textura; para la descripción de la morfología celular se empleó como colorante básico el azul de lactofenol mediante el método de la impronta con cinta transparente (Valencia, 2004).

Determinación de la abundancia relativa de las bacterias aisladas: Los microorganismos se clasificaron en categorías con base en la frecuencia de aparición según el criterio propuesto por Yadav y Madelin (Villafañe et al, 2009).

Análisis estadístico: Las diferencias de los tamaños poblacionales entre muestreos y entre parcelas se establecieron mediante análisis estándar de la varianza (Statistica 12.0), previa transformación de los datos a $\log _{10}$. Para los casos en los que se encontraron diferencias significativas, se efectuó un test post hoc de Bonferroni.

\section{RESULTADOS Y ANÁLISIS}

En la figura 2, se observa que aunque los tamaños poblacionales de heterótrofas totales fueron más altos en la parcela 2 y los más bajos se presentaron en la parcela 1, no existe diferencia significativa ni entre parcelas ni entre épocas de muestreo ( $p>0,05)$ (Tabla 1). 


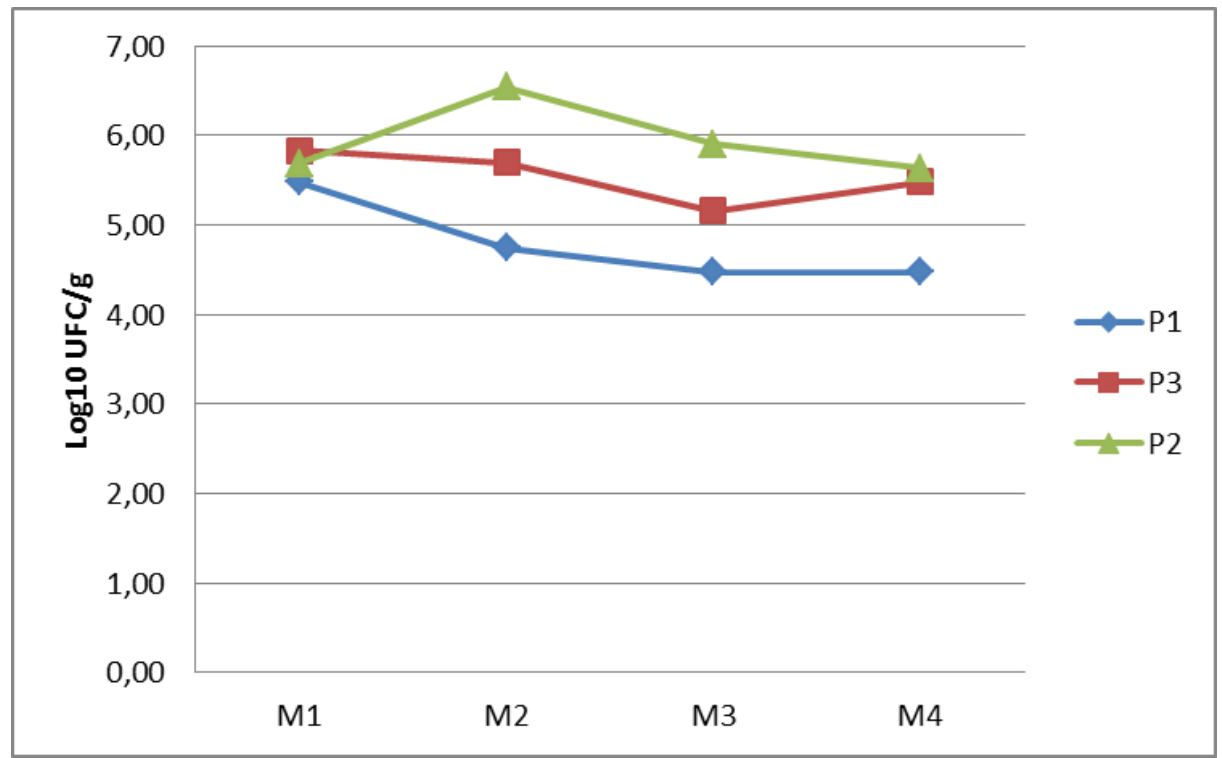

Figura 2. Tamaños poblacionales de heterótrofas totales en las tres parcelas durante las cuatro épocas de muestreo

Tabla 1. Análisis de varianza (ANOVA) efectuado a los grupos funcionales considerando diferencias entre épocas de muestreo y entre parcelas

\begin{tabular}{|c|c|c|}
\hline GRUPO FUNCIONAL & $\begin{array}{c}\text { POR } \\
\text { MUESTREO } \\
\text { (valor de p) }\end{array}$ & $\begin{array}{c}\text { POR PARCELA } \\
\text { (valor de p) }\end{array}$ \\
\hline Heterótrofas totales & 0,6790 & 0,1037 \\
\hline Amilolíticos & $\mathbf{0 , 0 0 0 9}$ & 0,7547 \\
\hline Fijadores de nitrógeno (Burk) & $\mathbf{0 , 0 1 0 9}$ & 0,4896 \\
\hline Nfb & 0,7056 & 0,8970 \\
\hline Hongos & 0,5532 & 0,1037 \\
\hline
\end{tabular}

Respecto a los microorganismos amilolíticos, la figura 3 muestra que los tamaños poblacionales más altos se presentaron en la parcela 3 y los más bajos en la parcela 2; sin embargo, las diferencias significativas se encontraron entre épocas de muestreo $(p \leq 0,05)$ y no entre parcelas $(p>0,05)$ (Tabla 1$)$, observándose que aunque los tres primeros muestreos corresponden a la temporada de lluvias los tamaños poblacionales entre ellos son diferentes y tiende a presentarse similitud entre periodos climáticos diferentes. 


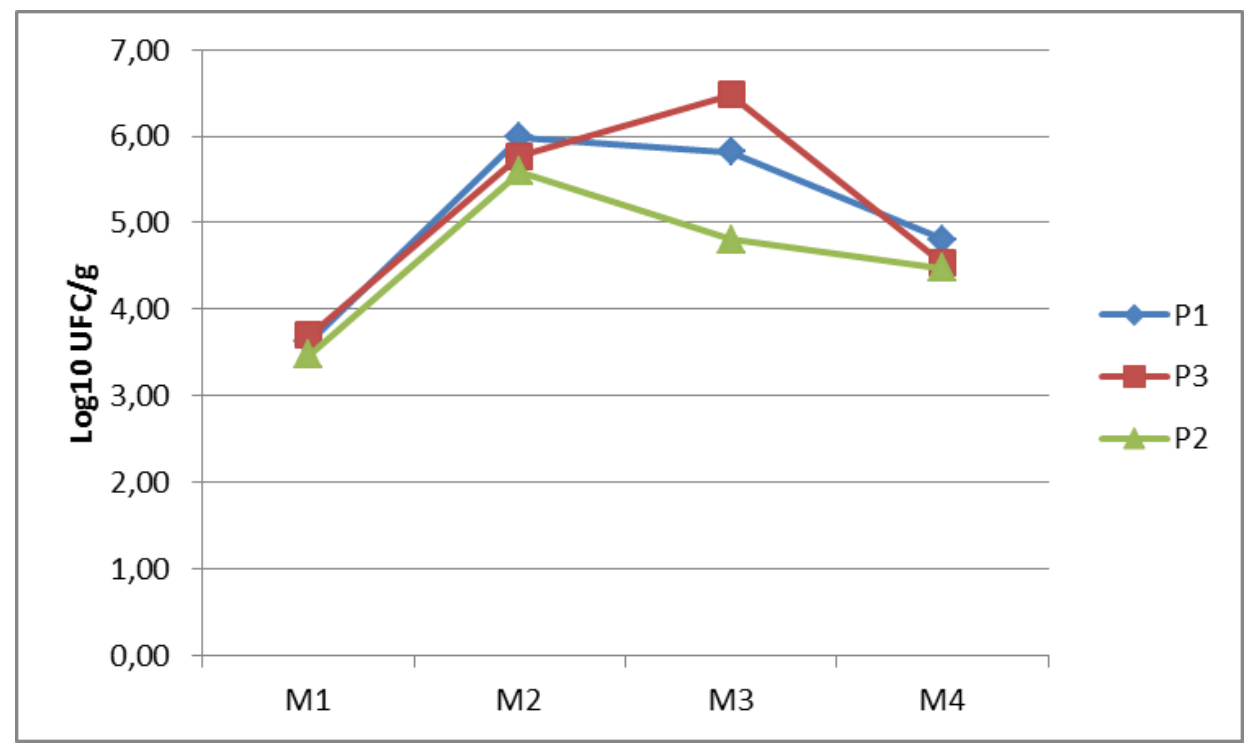

Figura 3. Tamaños poblacionales de microorganismos amilolíticos en las tres parcelas durante las cuatro épocas de muestreo

Los resultados del test post hoc de Bonferroni indicaron que las diferencias de tamaño poblacional se presentaron entre el muestreo 1 y los muestreos 2 y 3 (valores de $\mathrm{p} \leq 0,05$ ) (Tabla 2), observándose que el valor medio del tamaño poblacional registrado para el muestreo 1 es mucho menor $(3,5998)$ que el valor medio de los muestreos 2 y 3 (5,7844 y 5,6987, respectivamente), mientras que durante el muestreo 4 el valor fue ligeramente mayor al muestreo 1 , pero no estadísticamente diferente de éste.

Este resultado se debe a la transición que se presenta entre el segundo periodo seco del año que abarca los meses de junio a septiembre y el comienzo de la segunda temporada de lluvias que inicia en el mes de octubre; durante los siguientes meses que se acentúan las lluvias, el número de microorganismos aumentó.

Tabla 2. Resultados del test post hoc de Bonferroni efectuado al grupo funcional de amilolíticos

\begin{tabular}{|c|r|r|r|r|}
\hline \multirow{2}{*}{ Muestreo } & $\mathbf{1}$ & $\mathbf{2}$ & $\mathbf{3}$ & \multicolumn{1}{c|}{$\mathbf{4}$} \\
\cline { 2 - 5 } & & media $=$ & media $=$ & media $=$ \\
& media= 3,5998 & 5,7844 & 5,6987 & 4,6049 \\
\hline $\mathbf{1}$ & & $\mathbf{0 , 0 0 1 9}$ & $\mathbf{0 , 0 0 2 5}$ & 0,1465 \\
\hline $\mathbf{2}$ & $\mathbf{0 , 0 0 1 9}$ & & 1,0000 & 0,0706 \\
\hline $\mathbf{3}$ & $\mathbf{0 , 0 0 2 5}$ & 1,0000 & & 0,1007 \\
\hline $\mathbf{4}$ & 0,1465 & 0,0706 & 0,1007 & \\
\hline
\end{tabular}

Aunque los tamaños poblacionales de los microorganismos amilolíticos, (grupo funcional del carbono), no difirieron entre parcelas, un estudio realizado por Beltrán y Lizarazo (2013) estableció que la mayor carga microbiana encontrada en el grupo de microorganismos amilolíticos respecto al grupo de celulolíticos y proteolíticos dependió del tipo de material vegetal presente en el suelo; además, según Coyne (2000), los microorganismos descomponen el almidón con mayor facilidad que otros polisacáridos como la celulosa.

En un estudio realizado por Reyes et al (2011) con el fin de evaluar el efecto de la exposición y la toposecuencia sobre la actividad microbiológica en un relicto de bosque durante las dos estaciones del año se encontró que dicha actividad fue significativamente mayor durante el invierno y que tanto la exposición como los niveles toposecuenciales del terreno (ladera alta, ladera media y ladera baja) afectaron la actividad de los 
microorganismos. En este estudio, los tamaños poblacionales de microorganismos amilolíticos fueron más altos en los meses de lluvias (diciembre y abril) y la parcela 3, ubicada en la parte baja de la microcuenca, presentó los tamaños poblacionales más altos a pesar de no existir diferencia significativa con los tamaños de las otras dos parcelas.

Los tamaños poblacionales de fijadores de nitrógeno en el medio en el que estos microorganismos utilizan glucosa como fuente de carbono fueron más altos en la parcela 1 y los más bajos en la parcela 3 (Figura 4); las diferencias significativas se presentaron sólo entre épocas de muestreo (Tabla 1).

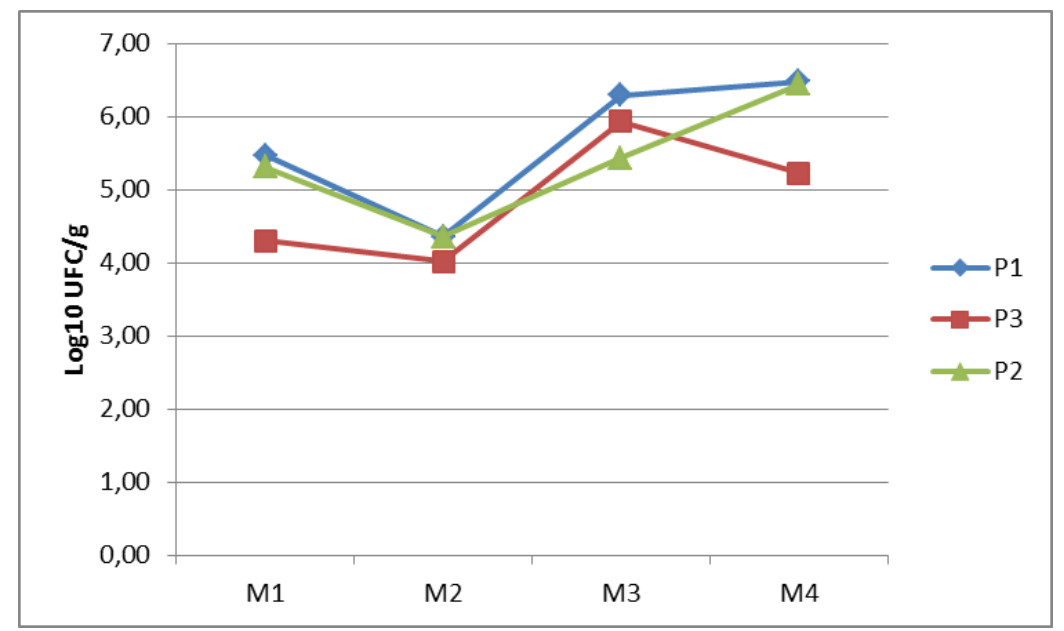

Figura 4. Tamaños poblacionales de bacterias fijadoras de nitrógeno (Burk) en las tres parcelas durante las cuatro épocas de muestreo

Los tamaños poblacionales de fijadores de nitrógeno en el medio en el que estos microorganismos utilizan malato como fuente de carbono fueron más altos en la parcela 2 y los más bajos en la parcela 3 (Figura 5); sin embargo, no existe diferencia significativa ni entre parcelas ni entre épocas de muestreo (p>0,05) (Tabla 1).

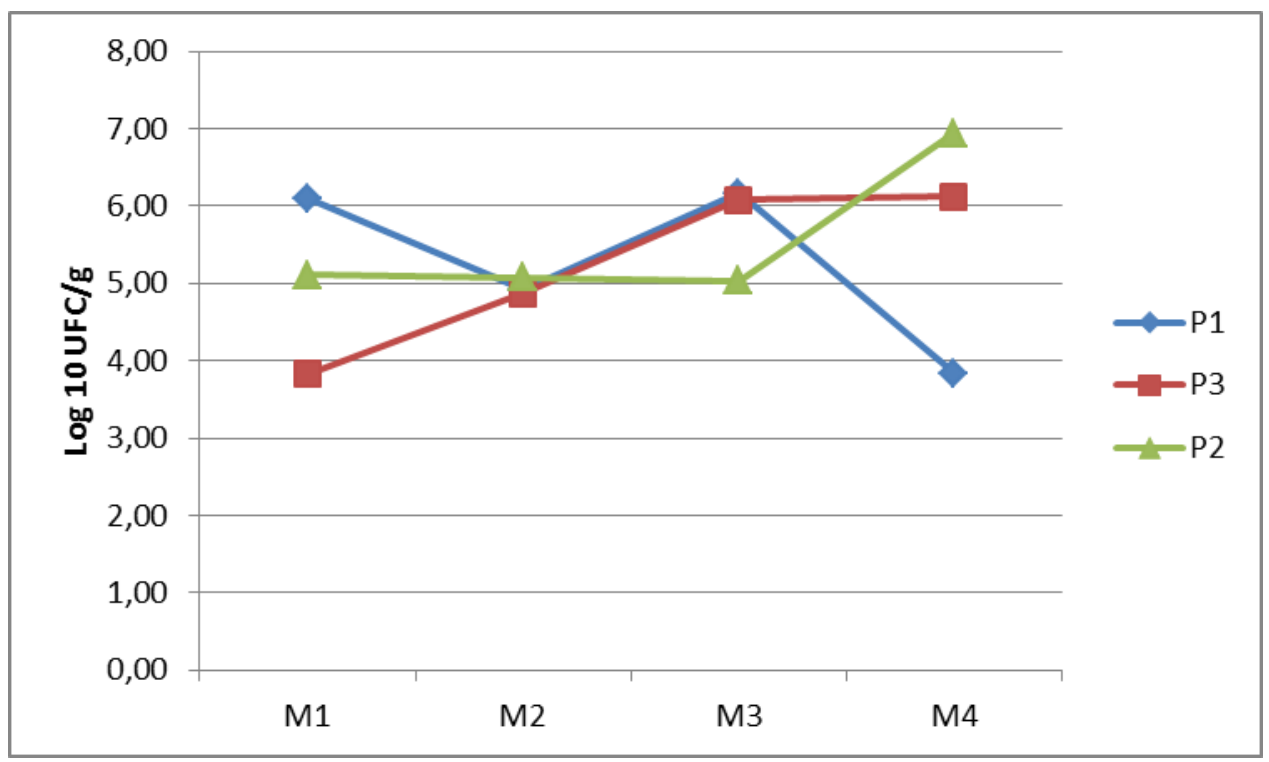

Figura 5. Tamaños poblacionales de bacterias fijadoras de nitrógeno $(\mathrm{Nfb})$ en las tres parcelas durante las cuatro épocas de muestreo 
De acuerdo con la tabla 3, los resultados del test post hoc Bonferroni indicaron diferencias de tamaño poblacional para fijadores de nitrógeno, según Burk, entre el muestreo 2 y los muestreos 3 y 4 (valores de $\mathrm{p} \leq$ 0,05) (Tabla 3), observando que durante el muestreo 2 la media del tamaño poblacional $(4,2457)$ fue inferior a los otros muestreos, aunque estadísticamente sólo difiere de los muestreos 3 y 4 (promedios 5,8860 y 6,0488, respectivamente).

Tabla 3. Resultados del test post hoc de Bonferroni efectuado al grupo funcional de fijadores de nitrógeno según Burk

\begin{tabular}{|c|r|r|r|r|}
\hline \multirow{2}{*}{ Muestreo } & $\mathbf{1}$ & $\mathbf{2}$ & $\mathbf{3}$ & \multicolumn{1}{c|}{$\mathbf{4}$} \\
\cline { 2 - 5 } & & & & media $=$ \\
& media= 5,0259 & media= 4,2457 & media $=5,8860$ & 6,0488 \\
\hline $\mathbf{1}$ & & 0,6633 & 0,4999 & 0,2791 \\
\hline $\mathbf{2}$ & 0,6637 & & $\mathbf{0 , 0 3 2 7}$ & $\mathbf{0 , 0 1 9 4}$ \\
\hline $\mathbf{3}$ & 0,4999 & $\mathbf{0 , 0 3 2 7}$ & & 1,0000 \\
\hline $\mathbf{4}$ & 0,2791 & $\mathbf{0 , 0 1 9 4}$ & 1,0000 & \\
\hline
\end{tabular}

La figura 6 muestra la relación entre tamaños poblacionales del grupo funcional amilolíticos y fijadores de nitrógeno según Burk en las diferentes épocas de muestreo poblacionales de amilolíticos (AMILOL) y de fijadores de nitrógeno (BURK) entre meses de muestreo. Las barras denotan valores máximos y mínimos.
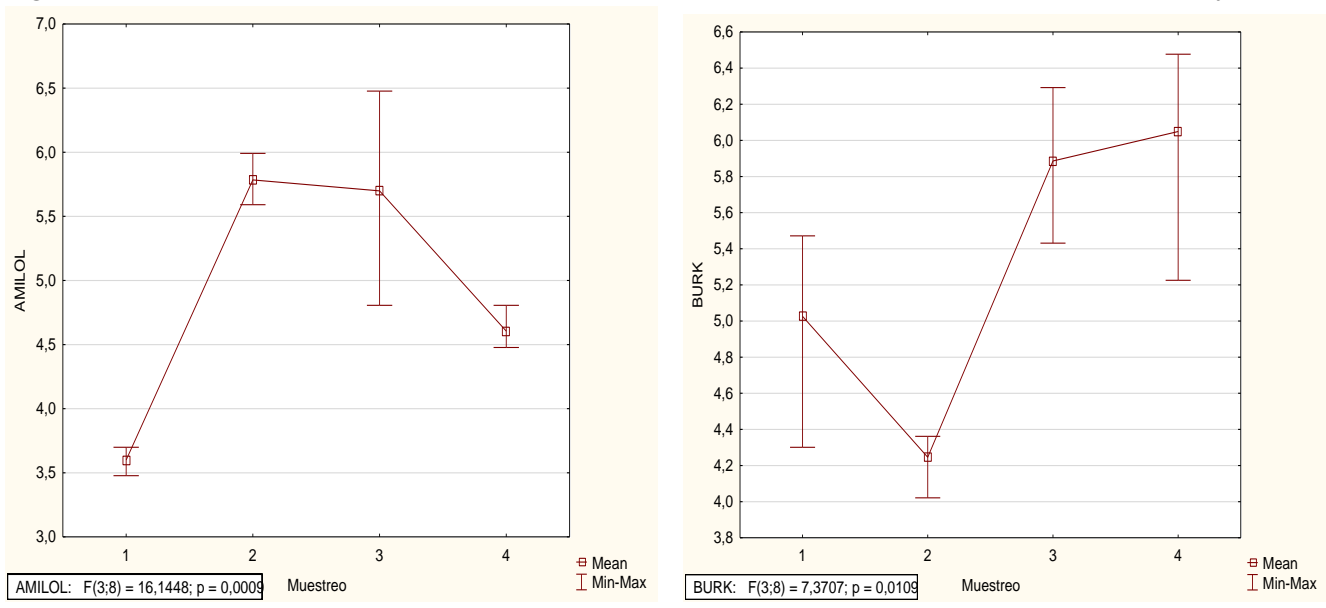

Figura 6. Variación temporal de los tamaños

Para el grupo funcional del nitrógeno los tamaños poblacionales más altos se observaron en el medio de cultivo donde los microorganismos utilizan glucosa como fuente de carbono $\left(6,48 \log _{10} \mathrm{UFC} / \mathrm{g}\right.$ de suelo) respecto al medio donde utilizan malato, lo cual según Beltrán y Lizarazo (2013) se debe a que en el suelo son más las fuentes naturales de glucosa que de malato, especialmente las que provienen del material vegetal.

Para el medio de Burk, las diferencias significativas encontradas entre las épocas de muestreo: el valor más alto durante la época seca respecto a las temporadas de lluvia, se puede explicar al hecho de que algunos de los microorganismos que realizan la fijación biológica del nitrógeno en el suelo desarrollan estructuras de resistencia como las endosporas para sobrevivir a condiciones de sequedad; las bacterias más abundantes caracterizadas en este estudio, luego del recuento de heterótrofas totales, correspondió a los bacilos grampositivos esporulados que presentan esta propiedad. 
Aunque la fijación biológica del nitrógeno realizada por los microorganismos de vida libre en el suelo demanda gran cantidad de energía química, su estudio cobra gran importancia en suelos boscosos donde los niveles de materia orgánica también son elevados.

La figura 7 revela que el tamaño poblacional más alto en el grupo de los hongos se presentó en la parcela 1 y el más bajo se presentó en la parcela 2; sin embargo, las diferencias no son significativas ni entre parcelas ni entre épocas de muestreo ( $p>0,05)$ (Tabla 1$)$.

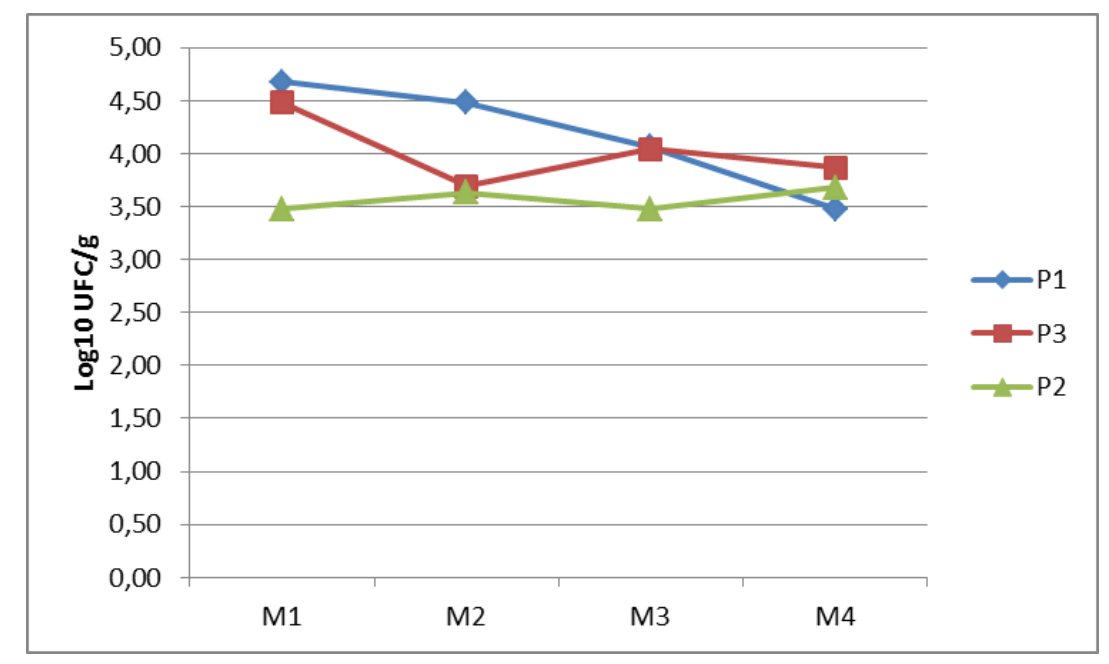

Figura 7. Tamaños poblacionales de hongos totales en las tres parcelas durante las cuatro temporadas del año

Respecto a los tamaños poblacionales de hongos, el valor más alto fue encontrado durante la temporada de lluvias, a pesar de no ser significativamente diferente a los valores de la temporada seca. De acuerdo con Atlas y Bartha (2008) el metabolismo de los hongos es activo cuando las condiciones de humedad y aireación son adecuadas y cunado existe un alto contenido de materia orgánica disponible para su utilización. Durante las lluvias, se acumula en el suelo material vegetal constituido, principalmente, por polisacáridos estructurales como la celulosa que los hongos hidrolizan con facilidad.

Los géneros fúngicos identificados en este estudio correspondieron a Paecilomyces sp., Aspergillus spp., Penicillium sp., Chrysosporium sp. y Acremonium sp. De acuerdo con Mayea et al y Giri et al (citado en Arias y Piñeros, 2008) los géneros de hongos más comunes en el suelo son Aspergillus, Acremonium, Botrytis, Cephalosporium, Penicillium, Trichoderma, Alternaria y Cladosporium, entre otros.

En otras investigaciones, se han aislado del suelo de bosque seco tropical hongos pertenecientes a los géneros Aspergillus sp., Beauveria sp., Cladosporium sp., Colletotrichum sp., Penicillium sp., Sclerotium sp., Tilletiopsis sp., y Trichoderma sp. (Pineda et al, 2009).

Teniendo en cuenta el criterio de clasificación de Yadav y Madelin (citado en Villafañe et al, 2009) los bacilos grampositivos esporulados fueron los más abundantes, presentando frecuencias de aparición hasta del $100 \%$ (parcela 2), mientras que los cocos gramnegativos fueron los menos abundantes y en dos de las parcelas no se aislaron (Tabla 4). 
Tabla 4. Abundancia relativa de los morfotipos bacterianos aislados, según clasificación de Yadav y Madelin

\begin{tabular}{|c|c|c|c|c|c|c|c|c|c|}
\hline & \multicolumn{3}{|c|}{ PARCELA 1} & \multicolumn{3}{|c|}{ PARCELA 2} & \multicolumn{3}{|c|}{ PARCELA 3} \\
\hline & $\mathbf{N}$ & $\%$ & $*$ & $\mathbf{N}$ & $\%$ & $*$ & $\mathbf{N}$ & $\%$ & $*$ \\
\hline Bacilos & & & & & & & & & \\
\hline Gramnegativos & 1 & 12,5 & $\mathrm{R}$ & 4 & 50,0 & $\mathrm{~F}$ & 2 & 25,0 & $\mathrm{O}$ \\
\hline Bacilos & & & & & & & & & \\
\hline $\begin{array}{l}\text { Grampositivos } \\
\text { esporulados }\end{array}$ & 7 & 87,5 & $\mathrm{MC}$ & 8 & 100,0 & $\mathrm{MC}$ & 5 & 62,5 & $\mathrm{C}$ \\
\hline Bacilos & & & & & & & & & \\
\hline $\begin{array}{l}\text { Grampositivos } \\
\text { no esporulados }\end{array}$ & 5 & 62,5 & $\mathrm{C}$ & 7 & 87,5 & $\mathrm{MC}$ & 6 & 75,0 & $\mathrm{C}$ \\
\hline Cocos & & & & & & & & & \\
\hline Gramnegativos & 1 & 12,5 & $\mathrm{R}$ & 0 & 0,0 & $\mathrm{NE}$ & 0 & 0,0 & $\mathrm{NE}$ \\
\hline Cocos & & & & & & & & & \\
\hline Grampositivos & 6 & 75,0 & $\mathrm{C}$ & 3 & 37,5 & $\mathrm{O}$ & 6 & 75,0 & $\mathrm{C}$ \\
\hline TOTAL & 20 & - & & 22 & - & & 19 & - & \\
\hline
\end{tabular}

Este hallazgo se sustenta con los estudios de flora realizados en La Tribuna, en los cuales Dueñas (2010) reportó una variedad de especies vegetales, principalmente de la familia Leguminosae (28 especies pertenecientes a 22 géneros) seguida de las familias Malvaceae, Asteraceae, Poaceae, Euphorbiaceae, Melastomataceae y Sapindaceae, lo cual es característico de los bosques intervenidos y secundarios (Olaya y Gutiérrez, 2014).

Los bacilos grampositivos no esporulados aislados en esta investigación, además de realizar la fijación biológica del nitrógeno en el suelo (bacterias diazótrofas), se vinculan a la descomposición de compuestos orgánicos; tal es el caso, de especies del género Arthrobacter que degradan herbicidas, fenoles y otros compuestos orgánicos (Madigan et al, 2003); lo anterior, guarda relación con los resultados de este estudio en el que se encontró que los bacilos grampositivos no esporulados presentaron frecuencia de aparición común cuando se aislaron de la parcela 1, influenciada por el asentamiento humano San Francisco y en el cual se realizan actividades de explotación petrolera.

Respecto al grupo de los bacilos gramnegativos aislados, los principales géneros relacionados con la fijación biológica de nitrógeno son las especies del género Azotobacter y Pseudomonas; respecto a este último género, algunos estudios han reportado especies que degradan compuestos orgánicos complejos incluyendo hidrocarburos (Téllez et al, 2000 citado en Benavides et al, 2006).

\section{CONCLUSIONES}

Las actividades biológicas del suelo son consideradas altamente sensibles a los cambios en la calidad del suelo cuando en éste varían las condiciones medioambientales (Alvear et al, 2008); en esta investigación, se encontró que las diferencias significativas de los tamaños poblacionales de microorganismos fueron determinadas por la temporada de lluvias o la temporada seca, según el grupo funcional estudiado. 
La mayor abundancia de microorganismos encontrada en la parcela 2 guarda relación con las características del bosque secundario en donde se encuentra localizada. Wadsworth (2000) afirma que las especies vegetales de los bosques secundarios probablemente presenten raíces profundas y difusas que agrupa una reserva de nutrientes que, para este caso, puede ser utilizada por los microorganismos del suelo.

Los grupos funcionales del carbono y del nitrógeno analizados actúan de forma sinérgica determinando las transformaciones químicas de estos dos elementos en el suelo. A modo de recomendación, debe investigarse acerca de las bacterias nativas aisladas en este estudio con el fin de establecer sus posibles aplicaciones biotecnológicas para el tratamiento del suelo por biorremediación.

\section{AGRADECIMIENTOS}

A la Vicerrectoría de Investigaciones y Proyección Social de la Universidad Surcolombiana por la financiación del proyecto.

\section{BIBLIOGRAFÍA}

Alvear, M., Astorga, M., Reyes, F. J. (2008). Efecto de los residuos vegetales provenientes de dos tratamientos silvícolas en una plantación de Pinus radiata D. Don sobre los cambios estacionales de las actividades biológicas del suelo. Soil Sc. Plant Nutr. 8 (1)

Arias, E y Piñeros, P. (2008). Aislamiento e identificación de hongos filamentosos de muestras de suelo de los páramos de Guasca y Cruz Verde. Facultad de Ciencias, Pontificia Universidad Javeriana

Atlas, R y Bartha, R. (2008). Ecología microbiana y Microbiología ambiental. $4^{\text {a }}$ edición. Pearson editores

Beltrán, M. y Lizarazo, L. (2013) Grupos funcionales de microorganismos en suelos de páramo perturbados por incendios forestales. Revista de Ciencias Facultad de Ciencias Naturales y Exactas, Universidad del Valle. Volumen $17 \mathrm{~N}^{\circ} 2$

Benavides, J., Quintero, G., Guevara, A., Jaimes, D., Gutiérrez, S., Miranda, J. (2006). Biorremediación de suelos contaminados con hidrocarburos derivados del petróleo. Revista Nova: Publicación científica en ciencias biomédicas. 4 (5)

Coyne, M. (2000). Microbiología del Suelo: un enfoque exploratorio. Editorial Paraninfo, España

Döbereiner. J. and Day. J. (1976). Associative symbiosis and free-living systems. In: Newton, W.E., Nyman, C.J., (eds.) Proceedings of the 1st International symposium on Nitrogen fixation. Washington State University. Press. Pullman. $518-538$

Dueñas, H. (2010). Flora del Centro de Investigaciones y Educación ambiental "La Tribuna" (Neiva, Huila, Colombia). La Tribuna: Reserva natural en zona petrolera del norte del Huila. Grupo de Investigación Ecosistemas Surcolombianos (ECOSURC). Universidad Surcolombiana-Ecopetrol. ISBN 978-958-8896-08-3

Esquivel, P., Mangiaterra, M., Giusiano, G., Sosa, M. (2003). Microhongos anemófilos en ambientes abiertos de dos ciudades del nordeste argentino. Boletín Micológico Vol. 18: 21- 28 DOI: http://dx.doi.org/10.22370/bolmicol.2003.18.0.376

Facultad de Ciencias Agropecuarias (2015). Guía de actividades prácticas Microbiología agrícola, Universidad Nacional de Córdoba 
Madigan, M., Martinko, J., Parker, J. (2003). Brock Biología de los microorganismos, 10ª ed. Pearson editores

Olaya, A. y Gutiérrez, G. (2014). La Tribuna: Reserva natural en zona petrolera del norte

del Huila. Grupo de Investigación Ecosistemas Surcolombianos (ECOSURC). Universidad Surcolombiana Ecopetrol. ISBN 978-958-8896-08-3

Pineda, M., Pineda, D., Labarca, J., Ulacio, D., Paredes, C., Casassa A. (2009). Microbiota del suelo asociada al cultivo del plátano (Musa AAB cv. Hartón) en bosque seco tropical del Sur del Lago de Maracaibo, Venezuela. Revista UDO Agrícola 9 (1)

Pizano, C. y García, H. (2014). El Bosque Seco Tropical en Colombia. Instituto de Investigación de Recursos Biológicos Alexander Von Humboldt

Pontecorvo, G., Roper, J., Hemons, L., Macdonalds, K. and Buffon, A. (1953). The genetics of Aspergillus nidulans. Adv. Gen 5: 141-238

Reyes, F., Lillo, A., Ojeda, N., Reyes, M., Alvear, M. (2011). Efecto de la exposición y la toposecuencia sobre actividades biológicas del suelo en bosque relicto del centro-sur de Chile. BOSQUE 32(3): 255-265 DOI: 10.4067/S0717-92002011000300007

Valencia, H. (2004). Manual de prácticas de Microbiología básica. Facultad de Ciencias, Universidad Nacional de Colombia

Valencia, H. (2010). Manual de prácticas de Microbiología del suelo. Facultad de Ciencias, Universidad Nacional de Colombia

Vargas, R., Cruz, A., Pedroza, Ch., Tovar, D., Pérez, C., Díaz, C. (2014). Caracterización geológica y de fluidos del Centro de Investigación "La Tribuna". La Tribuna: Reserva natural en zona petrolera del norte del Huila. Grupo de Investigación Ecosistemas Surcolombianos (ECOSURC). Universidad Surcolombiana -Ecopetrol. ISBN 978-958-8896-08-3

Villafañe,L., Castro, R., Castillo, D., Pinilla, M. (2009). Determinación de la carga fúngica anemófila en seis sectores de la ciudad de Cartagena de Indias. Ciencia y Salud Virtual 1(1)

Wadsworth, F. (2000). Producción forestal para América tropical: La América tropical, una región forestal. Departamento de agricultura de los Estados Unidos

Wilson, P. and Knight, S. (1952). Experiments in bacterial physiology. Burguess Publishing Co., Minneapolis. USA 